\title{
Role of inflammatory and synaptic plasticity genes in individual differences in cognitive abilities
}

Anastasiya Kazantseva

Institute of Biochemistry and Genetics

- Subdivision of the Ufa Federal

Research Centre of RAS, Ufa, Russia Kazantsa@mail.ru

Zalina Takhirova

Russian Academy of Education,

Moscow, Russia

Tahirovazalina@mail.ru

Marina Lobaskova

Psychological Institute, Russian

Academy of Education,

Moscow, Russia

Lobaskovamm@mail.ru
Renata Enikeeva

Institute of Biochemistry and Genetics

- Subdivision of the Ufa Federal

Research Centre of RAS, Ufa, Russia

Enikeevarf@gmail.com

Rustam Mustafin

Bashkir State Medical University, Ufa, Russia

Ruji79@mail.ru

Tatiana Tikhomirova

Russian Academy of Education, Moscow, Russia

Tikho@mail.ru
Yuliya Davydova

Institute of Biochemistry and Genetics

- Subdivision of the Ufa Federal

Research Centre of RAS, Ufa, Russia Julia.dmitrievna@list.ru

Sergey Malykh

Psychological Institute, Russian

Academy of Education,

Moscow, Russia

Malykhsb@mail.ru

Elza Khusnutdinova

Institute of Biochemistry and Genetics

- Subdivision of the Ufa Federal

Research Centre of RAS, Ufa, Russia

Elzakh@mail.ru

\begin{abstract}
The mechanisms underlying individual cognitive functioning are complex, and genetic and epigenetic factors play a significant role. Considering the possible involvement of inflammatory pathways and synaptic plasticity in cognitive functioning, the present study aimed to estimate the main effect of $B D N F, I L 1 B, C R P$, and $T N F$ gene polymorphisms together with gene-environment interactions in manifestation of individual differences in several cognitive abilities in young adults. Multiple linear regression conducted on the data obtained from 897 mentally healthy young adults (79\% women; $19.74 \pm 1.51$ years) of Caucasian origin revealed the association of $T N F$ rs1041981 A-allele and ability of 3D mental rotation, and general intelligence (factor "g"). Moreover, significant interactive effects of $T N F$ rs1041981 and $C R P$ rs3093077 and several peculiarities of childhood rearing on cognitive functioning were demonstrated.
\end{abstract}

Keywords - inflammation, cytokines, neurotrophic factor, gene association, cognitive abilities, number sense, general cognitive ability, working memory.

\section{Motivation and Aim}

\section{Motivation}

The study of the productivity of cognitive functions (the level of general intelligence, memory, spatial, mathematical, linguistic, etc. abilities) as an integral part of the individual personal potential is becoming increasingly relevant today, since the level of cognitive functioning is the basis of life success and individual self-realization. In particular, spatial ability, "number sense", general intelligence are predictors of success in life and professional activities, for example, in careers in science, technology, engineering and mathematics (STEM), as well as chemistry, physics, anatomy, psychology [1].

It is known that the mechanisms underlying individual predisposition to individual cognitive functioning are complex, and genetic and epigenetic factors play a significant role. Therefore, the manifestation of cognitive abilities is a very complex process, affecting the fine regulation of gene expression through epigenetic changes, as well as the modulation of the immune, endocrine and nervous systems through the involvement of metabolites in the functioning of brain systems. Together with the involvement of synaptic plasticity genes (including brain derived neurotrophic factor,
$B D N F$ ) in cognitive functioning [2], the pathways involved in immune function and inflammatory response, such as those responsible for cytokine and especially interleukin signalling while studying cognitive abilities/deficit are of great interest [3]. Namely, proinflammatory cytokines including interleukin 1 beta (IL1B) [4], C-reactive protein (CRP), tumor necrosis factor (TNF)) have been implicated in the pathogenesis of various psychiatric disorders; however, their role in cognitive functioning in mentally healthy individuals remains unsufficient. Multiple environmental factors may modulate gene-based inflammatory response via changes in individual epigenetic profile, thus resulting in individual differences in cognitive abilities.

Aim

In accordance with "Universalist genes hypothesis" and considering a role of proinflammatory cytokines and BDNF in cognitive impairment, the present study aimed to estimate the main effect of $B D N F, I L 1 B, C R P$, and $T N F$ gene polymorphisms together with gene-environment interactions in manifestation of individual differences in several cognitive abilities in young adults without cognitive deficit.

\section{Methods}

The study included 897 mentally healthy individuals (79\% women; $19.74 \pm 1.51$ years) of Caucasian origin (428 Russians, 200 Tatars, 117 Udmurts, and 152 of mixed ethnicity) from Russia. The assessment of cognitive abilities ("number sense", 3D mental rotation, general cognitive intelligence assessed with Raven's progressive matrices, Corsi blocktapping test for working memory) was conducted under the Battery of cognitive tests developed at International Laboratory for Interdisciplinary Investigations into Individual Differences in Learning (InLab) (Department of Psychology, Goldsmiths, University of London) and validated at the Laboratory of Age Psychogenetics (Psychological Institute, Russian Academy of Education, Moscow). The following environmental parameters, which were previously reported to affect cognitive functioning, were estimated: birth order, sibship size, place of residence, preterm birth, socio-economic status, rearing in a complete/incomplete family, childhood adversity, rearing in a bilingual family, smoking, physical activity, weight at birth, maternal and paternal age at birth. The genotyping of BDNF (rs6265), IL1B (rs16944), CRP 
(rs3093077), and TNF (rs1041981) gene SNPs was performed using PCR-based KASP genotyping technology on "CFX96" DNA Analyzer (BioRad, USA). Statistical analysis included multiple linear regression followed by FDR-correction for multiple testing (PLINK v.1.09). Genotypes and 13 environmental parameters served as independent factors and cognitive abilities as dependent variable.

\section{Results}

Statistical analysis revealed an association of TNF rs1041981 A-allele with increased ability of 3D mental rotation in total sample with sex and ethnicity inclusion as covariates $\left(\beta=1.61 ; \mathrm{P}=0.003 ; \mathrm{P}_{\mathrm{FDR}}=0.017\right)$ and among women $\left(\beta=1.57 ; \mathrm{P}=0.011 ; \mathrm{P}_{\mathrm{FDR}}=0.043\right)$. Interestingly, subsequent analysis of gene-by-environment interactions (GxE) demonstrated that place of residence (urban/rural status) modulated the association of TNF rs1041981 A-allele and the ability of $3 \mathrm{D}$ mental rotation $(\beta=-2.78 ; \mathrm{P}=0.020)$.

With respect to general intelligence (factor "g") we observed a sex-specific association of TNF rs1041981 Aallele with increased score on factor " $\mathrm{g}$ " only among women $\left(\beta=2.43 ; \quad \mathrm{P}=0.011 ; \quad \mathrm{P}_{\mathrm{FDR}}=0.045\right)$, while ethnicity-specific association was shown for $I L 1 B$ rs16944 A-allele and lower factor " $\mathrm{g}$ " in Tatars $\left(\beta=-5.57 ; \mathrm{P}=0.004 ; \mathrm{P}_{\mathrm{FDR}}=0.019\right)$. This finding seems to be congruent to those reported in elderly individuals without dementia [5]. It should be noted that GxE analysis demonstrated a modulating effect of bilingual rearing on the association of TNF rs1041981 A-allele and general intelligence $(\beta=5.33 ; \quad \mathrm{P}=0.015)$. Recently we also demonstrated sex- and ethnicity specific effect of synaptic plasticity genes polymorphisms in variation in such cognitive trait as mathematical anxiety [6].

The GxE analysis demonstrated that paternal age at individual's birth $(\beta=1.15 ; \mathrm{P}=0.008)$ and two-child family (sibship size $=2) \quad(\beta=-10.96 ; \quad \mathrm{P}=0.007)$ modulated the association of $C R P$ rs3093077 G-allele with "number sense", which reflects the ability to correctly place the number on the number-line. Recently, this SNP was proved in meta-analysis of depression [7], which evidence in the similar brain networks involved in depression and cognitive development.

In conclusion, the present preliminary study for the first time provides evidence that the genes involved in inflammatory pathways (TNF, CRP, and ILIB) may contribute to individual differences in such cognitive abilities as $3 \mathrm{D}$ mental rotation, general intelligence, and "number sense" in mentally healthy young adults. Moreover, our findings indicate significant interactive effects of examined gene polymorphisms and such environmental factors as place of residence, bilingual rearing, sibship size and paternal age at birth affecting cognitive functioning.

\section{ACKNOWLEDGMENT}

The present study was supported by the Russian Science Foundation (project No. 17-78-30028).

\section{REFERENCES}

[1] Nagy-Kondor R. (2017) Spatial ability of engineering students Annales Mathematicae et Informaticae. 34: 113-122.

[2] Toh Y.L. et al. (2018) Impact of brain-derived neurotrophic factor genetic polymorphism on cognition: A systematic review. Brain Behav. 8(7): e01009.

[3] McCall M.K. et al. (2018) Symptom Science: Omics Supports Common Biological Underpinnings Across Symptoms. Biol. Res. Nurs. 20(2): 183-191.

[4] Tsai S.J. (2017) Effects of interleukin-1beta polymorphisms on brain function and behavior in healthy and psychiatric disease conditions. Cytokine Growth Factor Rev. 37: 89-97.

[5] Sasayama D. et al. (2011) Association of interleukin-1 $\beta$ genetic polymorphisms with cognitive performance in elderly females without dementia. J. Hum. Genet. 56(8): 613-616.

[6] Kazantseva A. et al. (2020) Stress-associated cognitive functioning is controlled by variations in synaptic plasticity genes. Russian Journal of Genetics. 56 (1): 88-95.

[7] Cohen-Woods S. et al. (2018) Interaction between childhood maltreatment on immunogenetic risk in depression: Discovery and replication in clinical case-control samples. Brain Behav. Immun. 67: 203-210. 